\title{
Phase Detection Using Low-Loss EELS with Aberration Corrected STEM
}

\author{
C.T. Schamp, ${ }^{*}$ G. Casillas, ${ }^{* *}$ M. Jose-Yacaman, ${ }^{* *}$ \\ * SVTC Technologies, Austin, TX 78741 \\ ** Department of Physics and Astronomy, University of Texas at San Antonio, San Antonio, TX \\ 78249
}

The low-loss region of the electron energy loss spectrum (EELS) is dominated by the zero loss peak (ZLP), and peak intensities due to plasmon excitations. The width of the zero loss peak represents the energy spread of the system and its intensity is governed by the degree to which electrons were not inelastically scattered and were collected by the spectrometer (i.e. less counts means more inelastic scattering, more counts mean less inelastic scattering). The plasmon peaks are generally due to excitations of collective oscillations in the valence electrons of the sample [1]. In semiconducting materials, the valence electrons are highly localized and different phases can be readily mapped according to differences in plasmon energy, $E_{P}$. In metallic systems, the valence electrons are not as localized, and the "electron gas" will be strongly influenced by the presence of nearby phases so as to equilibrate Fermi levels, making differences in plasmon energies subtle near interfaces.

The core-shell-shell tri-metallic Pd-Au-Pd system creates a challenge for differentiating phases, particularly in the non-equilibrium kinetically trapped configuration of this structure. At equilibrium, the morphology may form an ordered structure, possess surface segregation effects, and in the nanoparticle system, may show unexpected phases, extended solubilities, or strong deviations from bulk lattice parameters [2-4]. Relatively straight-forward elemental linescans or maps are not necessarily sufficient to show phase distinction, although these techniques do show compositional variations. The low-loss region of the EEL spectrum has a very high signal-to-background ratio and can be used to distinguish the existence of unique phases along a linescan. Due to the inherent delocalization of the signal at low losses due to strong elastic tails, the difficulties of mapping metallic plasmons across interfaces are exasperated. To overcome this problem, inflection points in the smoothed linescan data are used here to determine interfaces between the core and shells.

$\mathrm{Pd}-\mathrm{Au}-\mathrm{Pd}$ core-shell-shell nanoparticles were prepared by successive alcoholic reduction on an electron transparent holey-carbon film supported on a copper mesh TEM grid for subsequent (S)TEM analysis. For further preparation details, see reference [5]. STEM-EELS analysis was carried out using the new JEOL ARM 200F CS probe corrected (S)TEM operated at 200kV at spot size $9 \mathrm{C}$ with a $\sim 34 \mathrm{mrad}$ convergence angle where the $40 \mu \mathrm{m}$ condenser aperture outline was fully enclosed within the flat part of the corrected Ronchigram. ADF-STEM images were recorded using the Gatan HAADF detector mounted directly above a Gatan Tridiem GIF. EELS data were recorded at a dispersion of $0.2 \mathrm{eV}$ per pixel. Low-loss EELS linescan data were recorded using a pixel spacing of $\sim 1 \AA$ across a line of about $10 \mathrm{~nm}$ through the center of a $\sim 8.5 \mathrm{~nm}$ Pd-Au-Pd nanoparticle. All acquisitions were through the Gatan Digital Micrograph software package.

The low loss EELS data were aligned according to the zero loss peak, then a variety of posprocessing techniques were applied to the dataset to optimize the dataset. These techniques include normalizing each spectrum by the area of the zero loss peak to reduce the impact of elastic 
scattering, normalizing each spectrum according to its relative thickness, and then deconvoluting each of the datasets using the Fourier-log deconvolution routine (raw, ZLP area normalized, and thickness normalized). The plasmon center energy for each dataset was determined by fitting a Gaussian function to the plasmon peak of each spectrum. All of this processing was performed within the Digital Micrograph Software package using built-in functions or custom scripts. The plasmon center energy data for each pixel in the linescan was then exported to the software package Origin for further processing. This additional processing involves smoothing through Fourier filtering using a 7 point window and then taking the second derivative of this smoothed dataset. Due to the band bending and the de-localization of the low-loss signal, the plasmon energy curve with position is a continuous function through the interfaces. However, at an interface, the rate of change in the slope will go to zero reflecting the transition in slope from one phase to another, e.g. an inflection point. The smoothed plasmon energy signal and the second derivative signal from the linescan across the particle in figure 1 are shown in figure 2, with the corresponding zero's mapped back onto the image.

Mapping the inflection points in the $\mathrm{E}_{\mathrm{P}}$ curve reveals boundary locations that are mostly consistent with the image with the subtle differences possibly representing the true three-dimensional structure.

\section{References}

[1] R.F. Egerton, Electron Energy-Loss Spectroscopy in the Electron Microscopy, Plenum, New York, 1996.

[2] Jesser, W.A. et al. Phys Rev B, 69(14): 144121 (2004).

[3] C.T. Schamp and W.A. Jesser. Met Trans A, 37A (2006) p.1825.

[4] Buffat, P. and Borel J.P. Phys Rev A 13(6): 2287 (1976).

[5] D. Ferrer, et al., Nano Lett. 2007, 7, 1701-170.

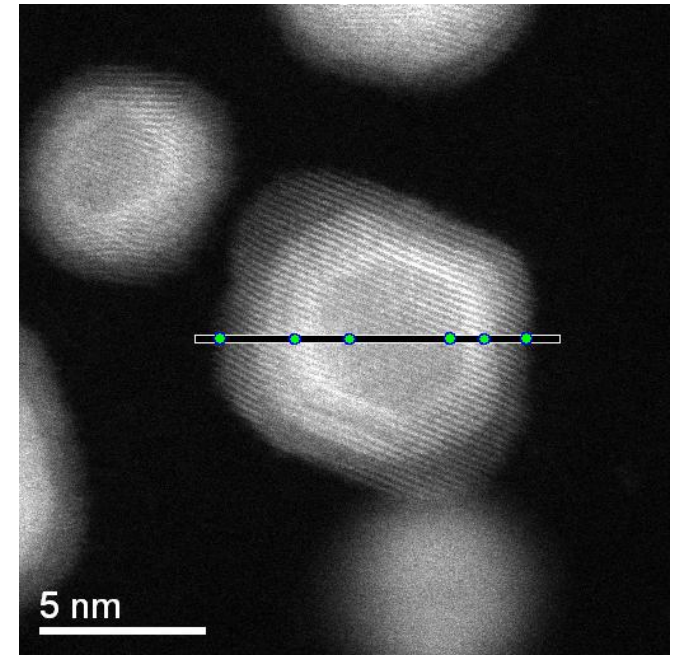

Figure 1. ADF-STEM image with EELS linescan location shown. Green circles indicate locations of inflection points in the $E_{P}$ curve.

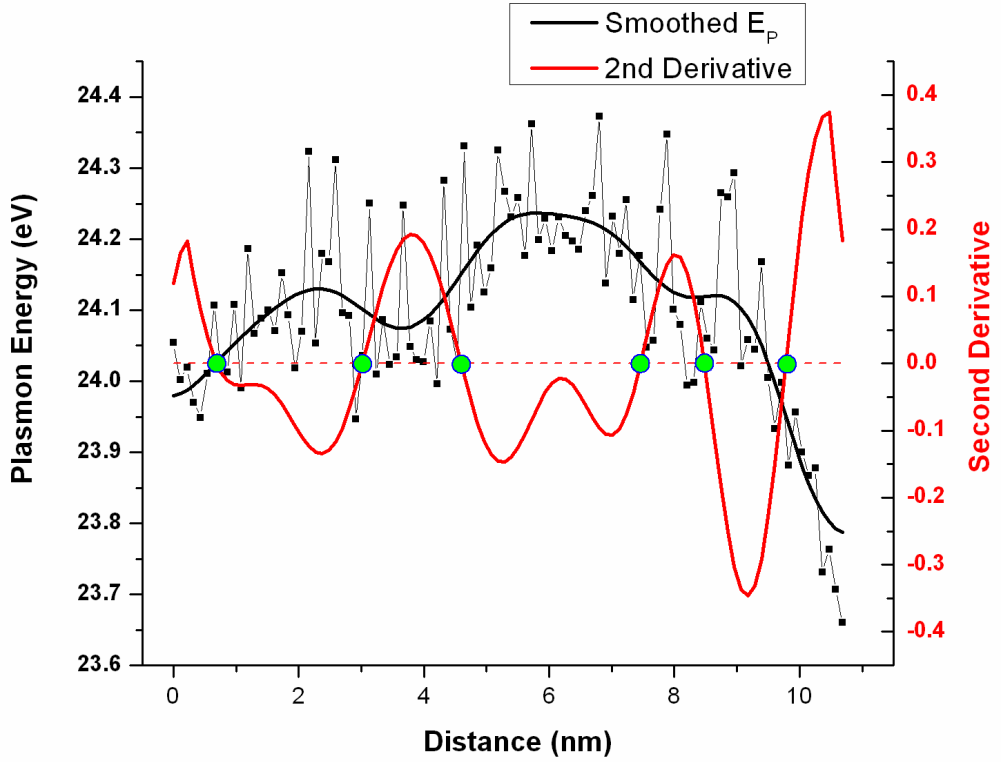

Figure 2. Noisy $E_{P}$ data smoothed with Fourier Filter (black curve). Second derivative of smoothed data shown in red with zeros (inflection points) shown in green and transferred to the corresponding locations on the image in figure 1. 